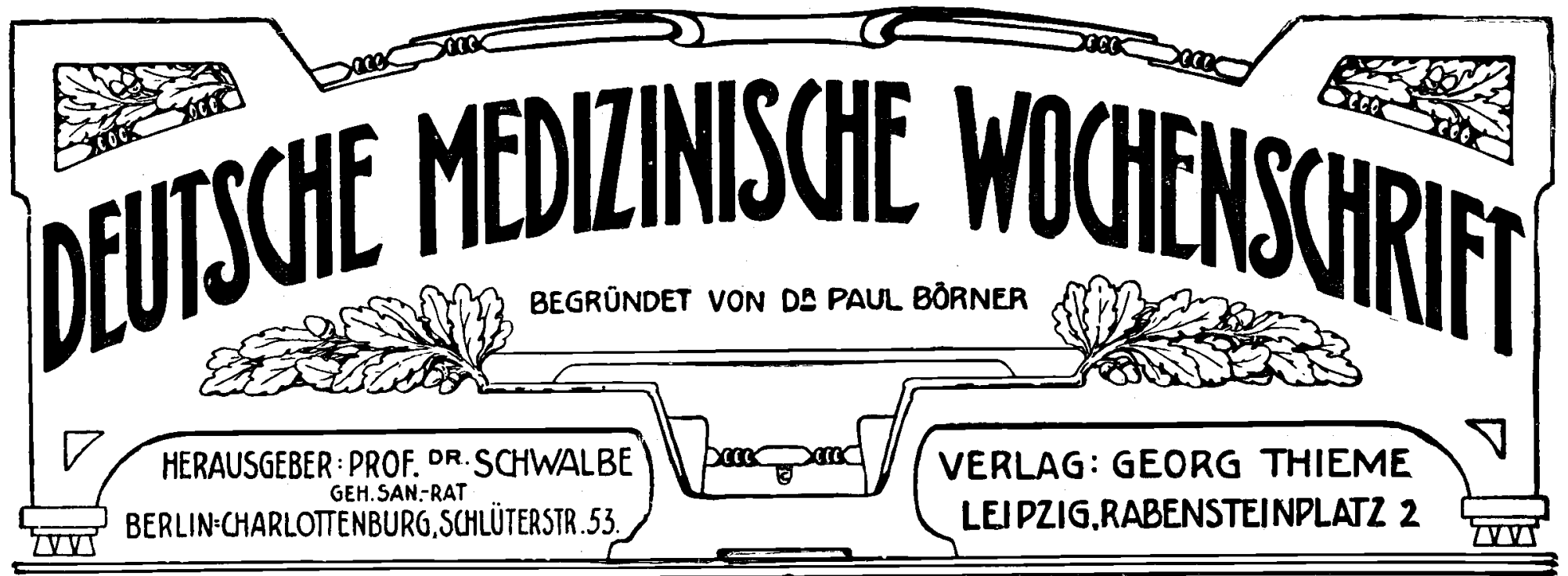

Nr. 43

BERLIN, DEN 24. OKTOBER 1912

38. JAHRGANG.

Aus dem Kgl. Institut für experimentelle Therapie in Frankfurt a. M. (Direktor: Wirkl. Geh. Rat Ehrlich.)

\title{
Erfahrungen über die Serodiagnostik der Syphilis.
}

Von H. Ritz und H. Sachs.

Trot $z$ der ausgezeichneten Erfolge, deren sich die Serodiagnostik der Syphilis mittels Wa.R. zu erfreuen hat, bestehen in den Erfahrungen verschiedener Untersuchungsstellen auch heute noch gewisse Differenzen, mit denen man allerdings nicht nur als mit einem gegebenen, sondern auch als mit einem durchaus verständlichen Faktor rechnen muß. Schon die Extraktfrage läßt es erklärlich erscheinen, daß gelegentlich die Resultate verschiedener Untersucher differieren können, ohne daß den einen oder den anderen ein Vorwurf zu treffen braucht. Dann aber haben sich auch in den verschiedenen Laboratorien bestimmte Gewohnheiten bei der Ausfiihrung der Reaktion herausgebildet, deren Unterschiede zwar im allgemeinen nicht wesentlich erscheinen, aber doch zu gewissen Widersprüchen führen können. So sind uns in mehrjähriger Erfahrung die sogenannten ,paradoxen“" Sera, die nach Meirowsky, M. Stern, Rasp und Sonntag u. a., bei wiederholter Untersuchung an mehreren Tagen regellos einmal positiv, einmal negativ reagieren können, nicht begegnet, und der eine von uns (Sachs) hatte vor kurzem auf der Tagung der südwestdeutschen Dermatologen in Frankfurt a. M. unsere negativen Befunde in dieser Richtung erwähnt. Das hat Meirowsky ${ }^{\mathbf{1}}$ ) Anlaß gegeben, die Frage der paradoxen Sera einer erneuten Besprechung zu unterziehen und dabei die Differenz, die in seinen und unseren Befunden besteht, auf die Verschiedenheit der von uns geübten Methodik zu beziehen, die nach dem genannten Autor ,,mit einem Ueberschuß von lösenden Faktoren arbeitet, der zwar keine paradoxen Sera aufkommen läßt, aber auch zahlreiche Sera mit geringem Gehalt an Syphilisstoffen der Diagnose durch die Wa.R. entzieht."

Wenn auch wir daher auf die sogenannten paradoxen Sera etwas näher eingehen dürfen, so scheint uns zunächst die Bezeichnung ,paradoxe Sera" nicht gliicklich gewählt. Denn wenn man mit Meirowsky und anderen Autoren den Wechsel der Reaktion als eine Funktion der Stärke von lösenden Faktoren auffaßt, so sind nicht die Sera paradox, sondern die Ergebnisse, und die Ursache hierfür liegt nicht in Veränderungen der Serunbeschaffenheit (vgl. auch Rasp und Sonntag), sondern in den Grenzen der Technik und Methodik, die eine absolute Identität der Versuchsbedingungen nicht ermöglichen. Nach unseren Erfahrungen kommt es wohl gelegentlich vor, daß die Reaktionsfähigkeit des Serums sich beim Lagern abschwächt, sodaß schwach positive Sera bei einer späteren Untersuchung dem Nachweis entgehen können; dagegen sind uns Sera, die zunächst negativ, später positiv reagieren, nicht begegnet. Aber gerade hierfür soll nun unser

3) Diese Wochenschrift Nr. 27 (1912).

nach Meirowsky zu stark gewähltes hämolytisches System verantwortlich sein. Es dürfte sich allerdings bereits bei theoretischer Ueberlegung nicht ohne weiteres ergeben, daß eine Verstärkung der Hämolyse paradoxe Reaktionen ausschalten muß. Wenn man nämlich die paradoxe Reaktion ergebenden Sera, wie es Meirowsky offenbar tut, als die am schwächsten positiv reagierenden auffaßt, so darf man doch wohl annehmen, $\mathrm{da} B$ bei der Untersuchung der verschiedenartigsten positiven Sera sich eine allmählich aufsteigende Skala von den am schwächsten bis zu den am stärksten reagierenden Serumproben ergibt. Wird demnach das hämolytische System vestärkt, so werden wohl die am geringsten positiv oder paradox reagierenden Sera negativ erscheinen, ebenso werden aber auch die übrigen Reaktionsgrade abgeschwächt werden. Es ist daher anzunehmen, daß auch bei einer Verstärkung des hämolytischen Systems paradoxe Reaktionen, wenn diese überhaupt zu den durch die Grenzen der Methodik bedingten Erscheinungsformen gehören, auftreten, nur daß eben hierbei eine andere Gruppe von Sera paradox reagiert.

Abgesehen von diesen Ausfïhrungen ist aber eine Gegenüberstellung der unserer Technik zugrunde liegenden Mengenverhältnisse und der von Meirowsky resp. in der Breslauer Dermatologischen Klinik benutzten Dosen der einzelnen Reagentien nicht hinreichend, um daraus den Schluß zu ziehen, da $B$ wir mit einem Ueberma $B$ von lösenden Faktoren arbeiten. Wir benutzen zwar nur die halben Serummengen $\left.(0,1 \mathrm{ccm})^{1}\right)$, dabei die doppelte Komplementdosis und eine etwas größere Ambozeptormenge, ${ }^{2}$ ) können aber trotzdem aussagen, daß ein Ueberma $B$ von lösenden Faktoren unter den bei uns obwaltenden Versuchsbedingungen nicht besteht. Die wesentliche Ursache, die einen Rückschluß aus den einfachen Zahlenangaben auf die relative Stärke des hämolytischen Systems nicht ohne weiteres zuläbt, ist in den Extrakten gelegen. Gerade die Verschiedenheit der Extrakte, mit denen an den einzelnen Instituten gearbeitet wird, läßt einen Vergleich absoluter Zahlenwerte recht illusorisch erscheinen. Von Bedeutung wäre dieser Vergleich nur unter gleichzeitiger Berücksichtigung der hämolytischen und eigenhemmenden Wirkung der Extrakte. Hier liegt aber der springende Punkt. Denn derjenige Faktor, der überhaupt dazu nötigt, ein mehrfaches Multiplum von Ambozeptoreinheiten für die Ausführung der Wa.R. zu verwenden, ist fast ausschließlich die eigenhemmende Extraktwirkung.

Wir stellen in dieser Hinsicht unsere Forderungen nicht mehr so rigoros, wie es bei Einführung der Methode üblich war, und verlangen nicht mehr, daß auch die doppelte Menge der Extraktgebrauchsdosis

1) Die Mengenangaben beziehen sich auf das Arbeiten mit $1 \mathrm{ccm}$ Hammelblutaufschwemmung. Tatsächlich sind im hiesigen Institut die Mengen aus ökonomischen Gründen bereits seit längerer Zeit auf den vierten Teil verringert, es handelt sich dabei aber, da sämtliche Komponenten die gleiche Reduktion erfahren, natürlich nư um rela. tive Werte.

t... - 2) Die Ambozeptordosis variiert in gewissen Grenzen, je nach der Beschaffenheit des Extraktes und Wirkungsart des Immunserums; in der Regel benutzen wir 4, zuweilen 3 Ambozeptoreinheiten. 
jeglicher antihämolytischer Wirkung entbehrt. Wir richten im Gegenteil unsere Versuchsanordnung so ein, daß die doppelte Extraktmenge eine mehr oder weniger starke Hemmung der Hämolyse (ohne Zusatz von Patientenserum) bedingt, weil wir hierdurch einen Indikator dafïr gewinnen, daß ein Uebermaß von lösenden Faktoren vermieden ist. Dagegen verlangen wir, daß diejenige Extraktmenge, bei deren Gebrauch wir eine positive Reaktion diagnostizieren, an und für sich jeglicher antikomplementären Wirkung entbehrt.

Die Anforderungen, die an die Extrakte gestellt werden, sind augenscheinlich nicht überall derartig. So glaubt Stern (Breslauer Methode) auf eine Kontrolle der hämolytischen Wirkung des Extraktes überhaupt verzichten zu können. Verzichtet man aber auf die Kenntnis der hämolytischen Extraktwirkung, so ist auch eine strenge Beurteilung der antikomplementären Eigenschaften kaum möglich. Es besteht anderseits wohl kein Zweifel, daß bei Verwendung von größeren, an und für sich (d. h. im Verein mit Meerschweinchenserum) hämolytisch wirkenden Extraktdosen die Reaktionsstärke bei syphilitischen Sera höher wird. Wir ziehen es aber: vor, uns mit geringeren Extraktmengen zu begnügen, um derart die stetige Kontrolle über die Eigenhemmung des Extraktes niemals zu verlieren und un nicht durch nichtspezifische Reaktionen getäuscht zu werden. ${ }^{1}$ )

Daß natürlich Schwankungen in der Lösungskraft des zur Verwendung gelangenden hämolytischen Systems vorkommen, ist durch die Variabilität der Meerschweinchensera und der Hammelblutkörperchen verständlich. Sie bewegen sich aber nach unseren Erfahrungen doch nur in gewissen Grenzen und stören die Beurteilung überhaupt kaum, wenn man sich nicht mit einer Ablesung der Ergebnisse nach zweistündigem Aufenthalt im Brutschrank oder nach einem noch größeren Intervall begnügt, sondern, wie es im hiesigen Institut die Regel ist, die Reaktion zeitlich verfolgt und unter Umständen bereits beurteilt, sobald die Kontrollen es ermöglichen. Soweit wir uns entsinnen können, ist von unserer Seite gerade bei der gleichen Gelegenheit, bei der von uns über das Fehlen von paradoxen Reaktionen berichtet wurde, darauf hingewiesen worden, daß dies wohl durch die von uns geübte zeitliche Beobachtung bedingt sein könnte. Besitzt eben das hämolytische System einmal etwas stärkere Wirkung, sodaß nach dem Ablauf der Reaktion der Extrakt auch in den größeren Dosen gar keine Hemmung mehr zeigt, so sind wir in der Lage, die Beurteilung bereits zu einer Zeit vorzunehmen, zu der bei den großen Extraktdosen noch eine Hemmung der Hämolyse wahrzunehmen ist. Es kann vorkommen, daß bei späterer Besichtigung gewisser Serumreaktionen komplette Hämolyse eingetreten ist, während in einem früheren Stadium, in dem die Kontrollen bereits hinreichend gelöst waren, noeh eine Hemmung bestand. Wir glauben, daß derartige Fälle wohl wesentlich das Kontingent der von anderen Autoren beobachteten paradoxen Sera bedingen dürften. Bei dem großen Material, das uns zur Verfügung steht, kommen natürlich nicht allzu selten Sera vor, die durch eine nur partielle Hemmung das Urteil erheblich erschweren. Die Untersuchung derartiger Sera wird stets am darauffolgenden Tage wiederholt, und so erwünscht es für die Diagnose wäre, können wir uns doch keiner Fälle entsinnen, die bei wiederholter Untersuchung zu einem wesent?ich sichereren Urteil, insbesondere nach der positiven Seite, geführt hätten. Wir begnügen uns dann mit der Diagnose ,"zweifelhaft" und bemühen uns, eine neu gewonnene Serumprobe zur nochmaligen Untersuchung zu erhalten. Gerade solche zweifelhaften Fälle wären aber wohl geeignet, zu paradoxen Reaktionen zu führen.

Was nun die Auffassung Meirowskys anlangt, daßi unsere Versuchsanordnung einen auffallend hohen Prozentsatz von negativen Reaktionen ergibt, so fußt diese Beurteilung auf Zahlen, die von $H$ öhne aus dem hiesigen Institut in der ersten Hälfte des Jahres 1909 mitgeteilt wurden und sich wesentlich auf die Erfahrungen des Jahres 1908 und der ersten Monate 1909 beziehen. Wie wohl an den meisten Untersuchungsstellen, so haben sich natürlich auch in unserem Laboratorium die Versuchsbedingungen im Laufe der Jahre, teils durch die

1) Die Prüfungen auf hämolytische Extraktwirkung haben natürlich nicht mit dem Extrakt allein zu geschehen, sondern unter Zusatz derjenigen Meerschweinchenserummenge, die der Versuchsanordnung zugrunde liegt (in unserem Falle $0,1 \mathrm{ccm}$ ), da ja durch das Serum als solches die hämolytische Wirkung der Extraktlipoide herabgesetzt wird.
Gewinnung besser wirkender Extrakte, teils durch exakterc Beobachtung und durch veränderte Anforderungen an die Beurteilung, verbessert, und wir haben daher zur Ergänzung der von Meirowsky angeführten, drei bis vier Jahre alten Zahlen Höhnes ein neueres Untersuchungsmaterial geordnet, das etwa bis Mai 1911 zurückgreift. ${ }^{1}$ ) Wir lassen die in den verschiedenen Stadien der Syphilis von uns erhaltenen Ergebnisse in der nachstehenden Tabelle folgen ${ }^{2}$ ) und stellen ihnen die älteren Angaben $\mathrm{Höhnes} \mathrm{gegenüber.}$

\begin{tabular}{|c|c|c|c|}
\hline \multicolumn{4}{|c|}{ Tabelle 1 . } \\
\hline & $\begin{array}{l}\text { Zahl der unter- } \\
\text { suchten Falle }\end{array}$ & $\begin{array}{c}\text { Positive Reak- } \\
\text { tionen in } \%\end{array}$ & $\begin{array}{l}\text { Posit. Reaktionen } \\
\text { (\%) nach Höhne }\end{array}$ \\
\hline Lues I & . . 120 & 53,5 & 38,6 \\
\hline Lues II (unbehandelt) & 93 & 98,9 & 79,1 \\
\hline Lues II ${ }^{3}$ ) (insgesamt) & 330 & 95,75 & - \\
\hline Lues III ${ }^{4}$ ) & 179 & 63,7 & 63,6 \\
\hline Lues cerebrospinalis ") & 197 & 47,2 & 16,7 \\
\hline Lues latens & . 1540 & 45,5 & 31,3 \\
\hline Tabes & 243 & 69,5 & 60 \\
\hline Paralyse & 194 & 86,1 & 80 \\
\hline
\end{tabular}

3) Fälle mit manifesten Symptomen, aber ohne Rücksicht auf eine eingeleitete Behandlung.

$\left.{ }^{4}\right)$ Die überwiegende Mehrheit der unter tertiärer Lues zusammengefaßten Fälle stand unter spezifischer Behandlung. Bei einer großen Zahl lassen die Angaben keinen Schluß zu, ob zur Zeit der' Blutentnahme noch Erscheinungen bestanden oder nicht. Klinische Symptome wiesen nacl den ärztlichen Angaben sicher 97 der untersuchten Patienten auf: davon betrafen:

\begin{tabular}{|c|c|c|}
\hline Lues III der: & Zahl & $\begin{array}{l}\text { Positive Reak } \\
\text { tionen }(\%)\end{array}$ \\
\hline $\begin{array}{l}\text { Haut } \\
\text { Knochen } \\
\text { Halsorgane } \\
\text { Innere und Sinnescrgane }\end{array}$ & $\begin{array}{l}37 \\
18 \\
25 \\
17\end{array}$ & $\begin{array}{l}75,6 \\
83,3 \\
52 \\
52,9\end{array}$ \\
\hline
\end{tabular}

5) Davon waren 127 bereits behandelt, bei $\mathbf{5 8}$ Patienten waren uns Angaben über etwaige Behandlung nicht zugegangen.

Die vorstehende Uebersicht zeigt, daß die Häufigkeit der Wa.R. bei Syphilis und metasyphilitischen Erkrankungen seit der von $\mathrm{H}$ öhne vor $3 \frac{1}{2}$ Jahren aufgestellten Statistik im hiesigen Institut erheblich zugenommen hat. Die fast ausnahmslos positive Reaktion bei unbehandelten Fällen von sekundärer Syphilis (von 93 - als , unbehandelt" bezeichneten - Blutproben reagierte nur eine negativ) und die hohe Prozentzahl $(95,75 \%)$ positiver Reaktionen im Sekundärstadium überhaupt sprechen zudem am besten dafür, daß die von uns geïbte Ausführungsart den zu stellenden Ansprüchen gerecht wird. Demgegenüber möchten wir der geringeren Anzahl positiver Reaktionen bei tertiärer Lues, bei Lues cerebrospinalis und bei Paralyse keine große Bedeutung beimessen. Wir sind uns wohl bewußt, daß von anderen Autoren die Häufigkeitsziffern größer angegeben werden, glauben aber, bei Berücksichtigung der verschiedenartigen Zusammensetzung des uns zur Verfügung stehenden Materials, die von uns erhaltenen Prozentzahlen als hinreichend hohe betrachten zu müssen. So sind die Fälle von tertiärer Syphilis und von Lues cerebrospinalis, deren Blutserum von uns untersucht wurde, in der überwiegenden Mehrheit bereits behandelt, und wir können auf Grund der uns zugänglichen Angaben nur bei einem gewissen Teile der Fälle aussagen, daß zur Zeit der Blutentnahme noch manifeste Symptome bestanden. Zu berücksichtigen ist außerdem natürlich die Frage der klinischen Diagnose und wohl auch das Krankheitsstadium. Wir dürfen in dieser Hinsicht vielleicht erwähnen, daß sich bei isolierter Beurteilung des uns von einer klinischen Anstalt zugegangenen Materials (118 Fälle) 58,4\% positive Reaktionen bei Lues cerebrospinalis ergeben haben, während unser Gesamtmaterial (197 Fälle) nur 47,2\% positive Reaktionen aufweist. In ähnlicher Weise ergibt das ausschließlich für die hiesige Städtische Irrenanstalt von uns in den letzten drei Jahren untersuchte Material von Paralysefällen (100), wie Herr Dr. Kirch berg in einer demnächst an anderer Stelle erscheinenden Arbeit berichtet, 93\% positive Reaktionen, und unter etwa 50 uns in den letzten Jahren von der medizinischen Klinik des hiesigen Städtischen Krankenhauses zugegangenen Paralysefällen ist, wie uns Herr Dr. Dreyfus mitteilt, eine negative Reaktion überhaupt nicht vorgekommen,

1) Bei der Zusammenstellung erfreuten wir uns der Mitarbeit von Fräulein-Johanna Conradi.

$\left.{ }^{2}\right)$ Es handelt rich hierbei nur um einen geringen 'Teil unseres Gesamtmaterials, das sich überwiegend aus den verschiedensten Kranksamtmaterials, das sich voerwiegend aus den verschiedensten
heitsfällen mit Luesverdacht zusammensetzt (cf. Tabelle 2 ). 
während die hier berücksichtigten 194 Fälle nur $86,1 \%$ positive Reaktionen aufweisen. Jedenfalls sind uns klinisch mit Sicherheit als Paralyse angesprochene Fälle bekannt, die bei uns negativ reagiert haben, und wir können daher auf Grund unserer Erfahrungen nicht behaupten, daß Paralyse bei der Wa.R. immer positiv reagiert.

Wie schon erwähnt, setzt sich die Mehrheit des von uns untersuchten Materials aus den verschiedenartigsten Krankheitsfällen zusammen, in denen Lues differentialdiagnostisch in Betracht kommt, oder bei denen Verdacht auf eine stattgehabte Luesinfektion besteht. Wir haben gleichwohl in der folgenden Tabelle 2 auch die übrigen, während der gleichen Zeitperiode im hiesigen Institut ausgeführten Blutuntersuchungen zusammengestellt und müssen dabei nur von vornherein betonen, daß dieser Aufstellung immerhin nur beschränkter Wert zugesprochen werden kann.

Tabelle 2.

\begin{tabular}{|c|c|c|c|c|c|c|c|c|c|c|c|c|c|}
\hline & $\frac{\overrightarrow{2}}{\dot{\omega}}$ & & & & & & $\mathscr{E} \mathrm{g}$ & & & & $\begin{array}{l}\text { Ver } \\
\text { ver }\end{array}$ & $\begin{array}{l}\text { tige } \\
\text { An }\end{array}$ & $\begin{array}{l}\text { inisch } \\
\text { le mit } \\
\text { : }\end{array}$ \\
\hline & 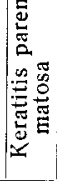 & 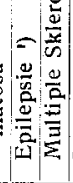 & $\mid \begin{array}{ll} & \\
\pi & 0 \\
\infty & 0 \\
0 & 0 \\
0 & 0 \\
0 & 0 \\
0 & 0 \\
0 & 0 \\
0 & 0\end{array}$ & 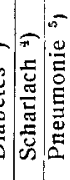 & 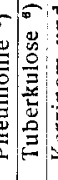 & 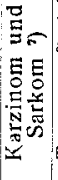 & 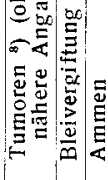 & 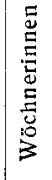 & 焉 & 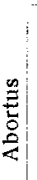 & 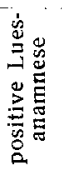 & 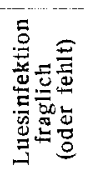 & 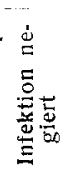 \\
\hline $\begin{array}{l}\text { esamtzahl } \\
\text { avon positiv }\end{array}$ & $\begin{array}{l}45 \\
34\end{array}$ & \begin{tabular}{r|r|}
39 & 22 \\
3 & 0
\end{tabular} & \begin{tabular}{|r|r}
14 & 23 \\
1 & 4
\end{tabular} & \begin{tabular}{r|r|r}
3 & 98 & 22 \\
4 & 3 & 5
\end{tabular} & $\begin{array}{l}269 \\
5 \\
5 \\
12\end{array}$ & $\begin{array}{r}54 \\
3\end{array}$ & \begin{tabular}{r|r}
80 & $23: 7$ \\
7 & 0
\end{tabular} & $\begin{array}{r}140 \\
28\end{array}$ & 38 & $\mid \begin{array}{c}30 \\
9\end{array}$ & $\begin{array}{l}482 \\
186\end{array}$ & $\begin{array}{r}2213 \\
461\end{array}$ & $\begin{array}{l}980 \\
130\end{array}$ \\
\hline oositiv in $\%$ & 75,5 & -- & & & & - & -- & & - & - & 38,5 & 20,8 & 13,2 \\
\hline
\end{tabular}

1) Bei 2 von den 3 positiven Fällen war die Luesanamnese positiv.

Bei dem positiv reagierenden Fall lag syphilitische Infektion vor.

$\left.{ }^{3}\right)$ Bei 3 von den 4 positiven Fällen Lues zugegeben. - 4 ) Bei 2 voll den 3 positiven Scharlachfällen bestanden Anhaltspunkte für Lues. - 5) Bei 4 von den 5 positiven Fällen Lues sicher, bei 1 Luesverdacht. - - Von den 12 positiven Fällen war bei 7 Luesinfektion angegeben, bei 2 bestand Luesverdacht. $\left.-{ }^{7}\right) 3$ positive Fälle; davon bei 2 Luesverdacht, 1 Puella publica. $-{ }^{8}$ ) Bei 4 von den 7 positiven Fällen fehlen Angaben über Lues; bei 3 Fällen Lues negiert.

Wenn wir in der Tabelle verschiedene Krankheitsformen herausgegriffen haben, so lag uns nicht wesentlich daran, neues Material für die heute allgemein anerkannte hohe diagnostische Bedeutung der Methode zu gewinnen. Daß wir einer Reihe von Blutproben begegnen, in denen trotz des Mangels von Angaben über klinische oder anamnestische Anhaltspunkte für Lues die Wa.R. positiv ausfällt, kann nicht überraschen, zumal ja in den uns zugehenden Fällen meist Luesverdacht besteht, und ein näheres Eingehen erübrigt sich um so mehr, als wir einem gehäuften Auftreten derartiger Fälle bei bestimmten Krankheitsformen nicht begegnet sind (cf. die Anmerkungen zu Tabelle 2). Wenn anderseits bei gewissen Gruppen (so bei Graviden und Wöchnerinnen) die Wa.R. in einer relativ hohen Zahl das Bestehen einer latenten Lues ergibt, so ist dabei auch wohl zu berücksichtigen, daß es sich ïberwiegend um Krankenhauspatienten handelt.

Einer besonderen Erwähnung ist aber vielleicht die oft diskutierte Scharlachfrage wert. Die drei positiven Reaktionen, die wir bei Untersuchung von 98 Scharlachpatienten antrafen, dürften allerdings als positive Scharlachreaktionen im Sinne von Much und Eichelberg nicht angesprochen werden.

Bei 2 dieser Scharlachkranken bestanden Anhaltspunkte, die einen Luesverdacht rechtfertigten, und es handelte sich in beiden Fällen um stärkste Reaktionsgrade, die auch bei mehrmaliger Untersuchung in Intervallen von 11 resp. 15 Tagen unverändert bestehen blieben. Der dritte Fall betrifft eine 30 jährige Patientin, bei der die zweimalig in 31 tägigen Intervallen vorgenommene Untersuchung jedenfalls übereinstimmend ein sehr stark positives Ergebnis lieferte. Wir sind daher geneigt, die positive Reaktion in diesen drei Fällen nicht auf Scharlach, sondern auf eine stattgehabte Luesinfektion zu beziehen.

Dagegen haben wir gerade in den letzten Wochen, nach AbschluB der statistischen Bearbeitung, nicht ganz selten Hemmungen bei Scharlach beobachtet. In fünf Fällen handelt es sich allerdings nur um partielle Hemmungen, die unter Berücksichtigung der Kontrollen nicht mehr als positiv, sondern nur als zweifelhafte Reaktionen bezeichnet werden konnten. In drei Fällen waren die Reaktionen aber derart, daß sie als schwach positiv gedeutet werden mußten, ohne daß irgendwelche Anhaltspunkte für Syphilis bestanden; und daB hier die Scharlachinfektion die positiven Reaktionen veranlaßte, darf man auch daraus schließen, daß bei einer späteren Untersuchung, die nach 10,17 resp. 26 Tagen vorgenommen wurde, die Hemmungen nicht bestanden oder dermaßen gering waren, daß sie nicht mehr im Sinne einer positiven Reaktion angesprochen werden konnten. Worauf das plötzliche häufigere Auftreten schwach positiver oder zweifelhafter Reaktionen bei Scharlach, die uns bisher unbekannt geblieben waren, zurückzuführen ist, müssen wir dahin gestellt sein lassen. Jedenfalls glauben wir danach auch auf Grund unserer Erfahrungen annehmen zu sollen, daß unter Umständen bei Scharlach schwach positive Reaktionen vorkommen können, ohne daß uns dadurch freilich die hohe diagnostische Bedeutung der Wa.R. beeinträchtigt erscheint.

Schließlich geben wir in der folgenden Tabelle noch eine kurze Uebersicht über die Ergebnisse der von uns im gleichen Zeitraum ausgeführten Untersuchungen von Lumbalflïssigkeiten.

Tabelle 3. Lumbalflüssigkeiten.

\begin{tabular}{|c|c|c|c|c|}
\hline & \multirow{2}{*}{$\begin{array}{c}\text { Zahl der } \\
\text { untersuchteı } \\
\text { Fälle }\end{array}$} & \multirow{2}{*}{$\begin{array}{c}\text { Positive } \\
\text { Reaktionent } \\
\%\end{array}$} & \multicolumn{2}{|c|}{$\begin{array}{c}\text { Auswertung } \\
\text { (Nonne-Hauptmann) }\end{array}$} \\
\hline & & & $\begin{array}{c}\text { Zahl der } \\
\text { Untersuchungen }\end{array}$ & $\begin{array}{c}\text { Fositiv } \\
\quad \%\end{array}$ \\
\hline 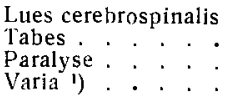 & $\begin{array}{r}120 \\
75 \\
92 \\
314\end{array}$ & $\begin{array}{l}40 \\
65,3 \\
76 \\
14,2\end{array}$ & $\begin{array}{l}49 \\
48 \\
29 \\
-\end{array}$ & $\begin{array}{l}55,1 \\
87,5 \\
79,3\end{array}$ \\
\hline
\end{tabular}

1) Bei den 49 positiv reagiecenden Fällen lauteten die ärztlichen Diagnosen, unter denen uns das Blut rugesendet wurde.

Lues cerebri?, Paralyse?, Tabes?

Lues (ohne nähere Angabe) .

Apoplexie

Meningitis, Neuritis acustica, Katatonie

Bemerkt sei, daß die Lumbalflüssigkeit in 19 Fällen von Epilepsi sowie in 11 Fällen von multipler sklerose negativ reagierte.

Bezüglich der bei der Untersuchung von Lumbalflüssigkeiten erhaltenen Zahlenwerte sind dieselben Bemerkungen an Platze, wie wir sie bei der Besprechung der Serumuntersuchungen gemacht haben. Die Auswertung der Lumbalflüssigkeit nach Nonne-Hauptmann, die wir jetzt ständig ausführen, haben wir erst im Frühjahr 1912 begonnen. Früher haben wir uns mit der Untersuchung der Lumbalflüssigkeiten in den Mengen von 0,2 bis höchstens $0,4 \mathrm{ccm}$ begnügt (auf $1 \mathrm{ccm}$ Blutaufschwemmung berechnet). Die Tabelle zeigt aber, dab auch wir durch die Anstellung von Reihenversuchen unter Verwendung von Lunıbalflüssigkeitsmengen bis $1 \mathrm{ccm}$ die Ergebnisse, insbesondere bei Lues cerebrospinalis und Tabes, nicht unerheblich verbessern konnten.

Trotz des großen uns zur Verfügung stehenden Materials und trotz zahlreicher wiederholter Untersuchungen ein und desselben Serums sind uns, wie schon erwähnt wurde, paradoxe Reaktionen nicht bekannt geworden, und wir glauben, daß hierbei die Art unserer Versuchsanordnung doch nicht ganz unbeteiligt ist. Insbesondere dürften dadurch, daß wir stets mit drei verschiedenen Extraktdosen arbeiten, von denen die größte die Grenze des Zulässigen fast erreicht, durch die zeitliche Beobachtung und eventuelle Beurteilung nach kurzer Frist Bedingungen gegeben sein, welche eine Paradoxie nicht zum Ausdruck gelangen lassen. Jedenfalls ergibt sich aus unseren Ausführungen, daß die Stärke des von uns benutzten hämolytischen Systems kaun für das Fehlen paradoxer Reaktionen verantwortlich gemacht werden kann. Eine Abschwächung der hänolytischell Kraft, im Sinne der von Meirowsky geübten Versuchsanordnung ist uns übrigens, wie vergleichende Untersuchungen von Schloss be rge r, über die später berichtet werden soll, zeigen, nicht immer ohne weiteres möglich gewesen. Wurde nämlich unter Verwendung der uns jetzt zur Verfügung stehenden Extrakte die Komplementmenge auf die Hälfte $(0,05 \mathrm{ccm})$ reduziert und nur mit drei Ambozeptoreinheiten gearbeitet, so traten eigenhemmende Wirkungen in den Kontrollen oft so erheblich in den Vordergrund, daß uns eine einwandfreie Beurteilung in vielen Fällen nicht mehr mög- 
lich war. ${ }^{\mathbf{1}}$ ) (Vergleiche hierzu auch Angaben von Kromayer und Trinchese.) Damit soll natürlich nicht ausgeschlossen werden, daß die Eigenhemmungen, soweit sie die Extrakte betreffen, bei Verwendung andersartiger Extrakte vermieden werden können und sich beim Arbeiten mit gewissen Extrakten Vorteile aus einer Reduktion der Konplementdosis ergeben. ${ }^{2}$ )

Wir sind fortgesetzt bemüht, durch Variation der Versuchsanordnung (insbesondere Aenderung der Serum- und Komplementdosen) unsere Methodik zu verbessern, sind allerdings, abgesehen von der sich auch weiterhin vorzüglich bewährenden Cholesterinierung von Rinderherzextrakten, vorläufig zu keinem uns praktisch wertvoll erscheinenden Fortschritt gelangt. Tmmerhin glauben wir uns mit den erzielten Ergebnissen, die fast durchweg die älteren Zahlen Höhnes mehr oder weniger erheblich übertreffen; durchaus begnügen zu dürfen und vorläufig Bedenken tragen zu müssen, durch eine uns nicht ungefährlich erscheinende Verfeinerung der Versuchsanordnung die Prozentzahl positiver Reaktionen bei sicheren Luesfällen zu steigern. 